\title{
Variance Estimation after Mass Imputation Based on Combined Administrative and Survey Data
}

\author{
Sander Scholtus ${ }^{1}$ and Jacco Daalmans ${ }^{1}$
}

\begin{abstract}
This article discusses methods for evaluating the variance of estimated frequency tables based on mass imputation. We consider a general set-up in which data may be available from both administrative sources and a sample survey. Mass imputation involves predicting the missing values of a target variable for the entire population. The motivating application for this article is the Dutch virtual population census, for which it has been proposed to use mass imputation to estimate tables involving educational attainment. We present a new analytical design-based variance estimator for a frequency table based on mass imputation. We also discuss a more general bootstrap method that can be used to estimate this variance. Both approaches are compared in a simulation study on artificial data and in an application to real data of the Dutch census of 2011.
\end{abstract}

Key words: Data integration; finite-population bootstrap; categorical data; census tables.

\section{Introduction}

Administrative data are being used ever more frequently in the production of official statistics (Bethlehem 2008). In many cases, available registers cannot meet all demands made by users of official statistics and therefore have to be supplemented by other data sources, most notably sample surveys. To ensure that the resulting statistics are of sufficient quality, it is necessary to evaluate their accuracy - in particular, their variances. In general, estimating the variance of an estimator based on combined administrative and survey data is not a trivial task.

As a running example, we consider variance estimation for frequency tables in the Dutch decennial virtual population census. Since 1981, the Dutch census tables have been estimated by re-using data from existing sources rather than collecting data with a dedicated questionnaire. Nowadays, most variables needed for the census are available from administrative sources with (near-)complete population coverage. An exception occurs for educational attainment, which is observed partly in education registers and partly in the Labour Force Survey (LFS). For about seven million Dutch persons (of a total of 17 million), educational attainment is not observed.

\footnotetext{
${ }^{1}$ Statistics Netherlands, Department of Process Development and Methodology, P.O. Box 24500, 2490 HA The Hague, the Netherlands. Emails: sshs@cbs.nl and jdas@cbs.nl

Acknowledgments: The views expressed in this article are those of the authors and do not necessarily reflect the policies of Statistics Netherlands. We would like to thank our colleagues Arnout van Delden, Jeroen Pannekoek, Eric Schulte Nordholt, and Ton de Waal for helpful discussions about this topic and for commenting on early versions of this article. We would also like to thank the Associate Editor and three anonymous referees for their review comments that have greatly improved this article.
} 
In the Dutch censuses of 2001 and 2011, the "repeated weighting method" was used to handle missing data during estimation of census tables (Schulte Nordholt et al. 2004, 2014). It is known that repeated weighting has practical limitations for the estimation of large numbers of high-dimensional frequency tables, and these limitations were indeed encountered during the 2011 census (Daalmans 2018). As an alternative approach, it has been proposed for the next census to use mass imputation to predict all missing values of educational attainment (De Waal et al. 2018). This naturally leads to the question how to determine the precision of estimated entries in frequency tables based on mass-imputed data.

In principle, one may try to use analytical approximations to estimate the variance of statistical tables after mass imputation and, by extension, other estimators based on combined administrative and survey data. For instance, Knottnerus and van Duin (2006) developed an analytical variance approximation for estimated census tables based on repeated weighting. For the particular case of estimated frequency tables, we will present a new formula for the design-based variance of mass-imputed estimates in Section 2. This formula is of interest for a class of problems in which a categorical variable is massimputed, using one or more auxiliary variables. It is assumed here that a certain model is applied that predicts, for each record, the probabilities for each of the categories of the target variable. For instance, this can be a logistic regression model. Imputations for missing values are then drawn based on these predicted probabilities.

In general, a drawback of analytical variance estimation is that a separate expression has to be derived for each estimator and each data configuration. In fact, for some estimators based on combined data, deriving an adequate variance expression may prove to be impossible. Therefore, a resampling method such as the bootstrap may be a more attractive option. In Section 3, a generic bootstrap method will be described for this purpose. Unlike the analytical approach of Section 2, this bootstrap approach does not depend on the specific context of mass imputation of a categorical variable and could therefore easily be applied to many other estimators based on combined administrative and survey data.

Both approaches have been tested on a small artificial population in a simulation study (Section 5). In Section 6, we will describe an application of both approaches to real data of the Dutch virtual census 2011. Both applications in Sections 5 and 6 make use of a specific imputation model that has been proposed for the next Dutch census, the "continuationratio model"; this model will be outlined in Section 4. In particular, we will present a new analytical expression for the large-sample variances of predicted probabilities based on this model. Some conclusions follow in Section 7.

\section{Analytical Variance Estimation for Mass-Imputed Tables}

We will now consider the estimation of frequency tables based on mass imputation in more detail. Let $\theta_{h c}=\sum_{i \in U} h_{i} y_{c i}$ denote the true count in a particular cell of a table involving our target variable. Here, $U$ is the target population and $y_{c}$ is an indicator variable such that $y_{c i}=1$ if person $i$ belongs to category $c$ of the target variable and $y_{c i}=0$ otherwise ( $c=1, \ldots, C$, where $C$ denotes the total number of categories). Furthermore, $h$ is a similar indicator variable for the cross-classification of all other variables in the table, that is, $h_{i}=1$ if person $i$ contributes to this cell according to these other variables and $h_{i}=0$ otherwise. 
As is common in official statistics, we will consider the target population and the values of variables for units in this population as fixed. All variables other than the target variable represented by $y_{1}, \ldots, y_{C}$ are supposed to be completely observed for all units in the population. The target variable is partially observed. In general, it could be observed for some units in an administrative source and for other units in a sample survey. We suppose that missing values on the target variable are imputed throughout the population (mass imputation; see, e.g., De Waal et al. 2011). After mass imputation, $\theta_{h c}$ is estimated as follows:

$$
\hat{\theta}_{h c}=\theta_{h c 1}+\hat{\theta}_{h c 2}=\sum_{i \in U_{1}} h_{i} y_{c i}+\left(\sum_{i \in S_{2}} h_{i} y_{c i}+\sum_{i \in U_{2} \backslash S_{2}} h_{i} \tilde{y}_{c i}\right) .
$$

Here, $U_{1}$ consists of all persons in $U$ for which the target variable is observed in a register. From the remaining subpopulation, $U_{2}=U \backslash U_{1}$, a probability sample $S_{2}$ is available with observed values of the target variable. Finally, for all $i \in U_{2} \backslash S_{2}$, the indicator $y_{c i}$ is unknown and replaced by an imputation $\tilde{y}_{c i}$ in Equation (1).

We will consider the register part of the population, $U_{1}$, to be fixed. In general, the sample $S_{2}$ may be a subsample of a sample $S$ drawn from $U$. Note that the size of the register overlap $S_{1}=S \cap U_{1}$ is then random in general.

In the application to be discussed in Section 6, Equation (1) is used to estimate a table in the Dutch virtual census, where $y_{1}, \ldots, y_{C}$ represent levels of educational attainment. In this case, the imputations are based on a model that is estimated only on data from $S_{2}$, as the register data are known to be selective. The results in this section can be applied to any frequency table involving a categorical variable that is mass-imputed. This includes the special case where only sample survey data are available $\left(U_{1}=\varnothing\right)$.

In general, we suppose that the missing values of $y_{c i}$ are imputed by drawing independently for each person $i \in U_{2} \backslash S_{2}-$ a vector $\left(\widetilde{y}_{1 i}, \ldots, \tilde{y}_{C i}\right)$ from a multinomial distribution with predicted probabilities $\left(\hat{p}_{1 i}, \ldots, \hat{p}_{C i}\right)$, so that for each $i$ exactly one of the values $\tilde{y}_{c i}$ is equal to 1 and the other values are equal to 0 . The predicted probabilities $\hat{p}_{c i}$ are obtained from an imputation model estimated on the observed distribution of $y_{c}$ in the sample $S_{2}$. An example of an imputation model will be discussed in Section 4.

The uncertainty in $\hat{\theta}_{h c}-\theta_{h c}$ comes from three different sources: the random selection of persons in $U_{2} \backslash S_{2}$ that have to be imputed, the uncertainty in the predicted probabilities $\hat{p}_{c i}$ for these persons, and finally the stochastic drawing of the imputations $\tilde{y}_{c i}$ based on these predicted probabilities. All of these uncertainties are directly or indirectly related to the selection of the probability sample $S_{2}$ and therefore depend on the design of this sample.

In principle, it is possible to evaluate the variance of $\hat{\theta}_{h c}-\theta_{h c}$ analytically. Here, we will make the simplifying assumption that the predicted probabilities $\hat{p}_{c i}$ in $S_{2}$ satisfy the following condition, for all $c=1, \ldots, C$ :

$$
\sum_{i \in S_{2}} h_{i}\left(\hat{p}_{c i}-y_{c i}\right)=0
$$


Under this assumption, the following variance formula is derived in Appendix (Subsection 8.1):

$$
\operatorname{var}\left\{\hat{\theta}_{h c}-\theta_{h c}\right\}=E\left\{\sum_{i \in U_{2} \backslash S_{2}} h_{i} \hat{p}_{c i}\left\{1-\hat{p}_{c i}\right\}\right\}+\sum_{i \in U_{2}} \sum_{j \in U_{2}} h_{i} h_{j} \operatorname{cov}\left\{\hat{p}_{c i}, \hat{p}_{c j}\right\} .
$$

For many commonly used models, Equation (2) may be reasonable, at least as an approximation. For instance, it is satisfied exactly when imputation is based on a logistic regression model, fitted by maximum likelihood to a simple random sample $S_{2}$, which includes the cross-classified variable $h$ as a predictor (see, e.g., Agresti 2013, 192-193). However, there are also many situations where Equation (2) will not hold exactly; for instance, if $S_{2}$ in the previous example has a complex survey design, the sampling weights are used when estimating the logistic regression model and these weights are not contained in the space spanned by the predictor variables. In Appendix (Subsection 8.1), it is argued that Equation (3) will be a reasonable approximation to the true variance even when Equation (2) does not hold, provided that the sampling fraction of $S_{2}$ from $U_{2}$ is sufficiently small (see the Appendix for details). In the next section, a bootstrap method will be described that can be used instead of Equation (3) if the use of Equation (2) is problematic.

To complete the specification of $\operatorname{var}\left(\hat{\theta}_{h c}-\theta_{h c}\right)$, an expression is needed for $\operatorname{cov}\left(\hat{p}_{c i}, \hat{p}_{c j}\right)$ in Equation (3). The precise form of this covariance depends on the imputation model and the sample design. For the proposed imputation model for educational attainment in the Dutch census, an approximate expression for $\operatorname{cov}\left(\hat{p}_{c i}, \hat{p}_{c j}\right)$ will be derived in Section 4 and Appendix (Subsection 8.2); there it is also discussed under which conditions Equation (2) holds (approximately) for this model. Evaluating the resulting variance formula may be computationally challenging in practice.

Based on the observed data, the variance in Equation (3) could be estimated as follows:

$$
\widehat{\operatorname{var}}\left(\hat{\theta}_{h c}-\theta_{h c}\right)=\sum_{i \in U_{2} \backslash S_{2}} h_{i} \hat{p}_{c i}\left(1-\hat{p}_{c i}\right)+\sum_{i \in U_{2}} \sum_{j \in U_{2}} h_{i} h_{j} \widehat{\operatorname{cov}}\left(\hat{p}_{c i}, \hat{p}_{c j}\right) .
$$

Here, $\widehat{\operatorname{cov}}\left(\hat{p}_{c i}, \hat{p}_{c j}\right)$ denotes an estimator of $\operatorname{cov}\left(\hat{p}_{c i}, \hat{p}_{c j}\right)$. Again, the precise form of this term depends on the imputation model and the sample design. Note that the first term in Equation (4) follows from the fact that any random variable is an unbiased estimator for its own expectation.

Note that in Scholtus (2018) an alternative variance estimator was derived, without Equation (2) but with the additional technical assumption that $\hat{p}_{c i}$ is stochastically independent of the sample inclusion indicator of $S_{2}$ for all units. Based on the simulation study presented there and in Section 5 of this article, it appears that this assumption can be failed in practice and as a result this alternative formula may underestimate the true variance. Note that this independence assumption is not easy to check based on the observed data, unlike Equation (2).

As far as we are aware, the above design-based variance of a mass-imputed estimator for categorical data has not been mentioned before in the literature. Valliant et al. (2000) discussed estimators of this type from a model-based perspective, but focused mainly on numerical target variables. Kim et al. (2020) consider variance estimation for a more general imputation model, for a situation that they call 'mass imputation' but which does not involve imputation of missing data throughout the target population. 


\section{A Bootstrap Method}

The classical bootstrap (Efron 1979; see, e.g., Efron and Tibshirani 1993, for an introduction) uses resampling with replacement from an original sample to approximate the sampling distribution of a target estimator. This method cannot be used directly here, as it does not account for finite-population sampling. In fact, mass imputation is meaningful only in the context of a finite population.

Different extensions of the bootstrap to finite-population sampling have been developed; see Mashreghi et al. (2016) for a recent overview. For estimators that involve weighting or imputation, a particularly useful extension is based on generating pseudopopulations. This methodology was developed by Gross (1980), Booth et al. (1994), Canty and Davison (1999), and Chauvet (2007). At Statistics Netherlands, Kuijvenhoven and Scholtus (2011) applied this type of bootstrap method to combined register and LFS data on educational attainment, for various estimators based on weighting. Here, we will describe a slight extension of their method that can accommodate more general estimators.

As a generalisation of Equation (1), suppose that the estimator of interest is $\hat{\theta}=t\left(S, U_{1}\right)$, for some functional $t\left(\right.$.). The underlying finite-population parameter is $\theta$. Let $\pi_{i}$ denote the inclusion probabilities of sample $S$, and write the design weights $w_{i}=1 / \pi_{i}$ as $w_{i}=\left\lfloor w_{i}\right\rfloor+\varphi_{i}$, with $\left\lfloor w_{i}\right\rfloor \in \mathbb{N}$ and $\varphi_{i} \in[0,1)$. Here, $\lfloor z\rfloor$ denotes the integer part of $z \in \mathbb{R}$, that is, the largest integer that is smaller than or equal to $z$. The bootstrap algorithm consists of the following steps:

- For each $a=1, \ldots, A$ do the following:

1. Create a pseudo-population $\hat{U}_{a}^{*}$ by taking $\omega_{i}$ copies of each unit $i \in S$, where the random inflation weight $\omega_{i}$ is chosen to be $\omega_{i}=\left\lfloor w_{i}\right\rfloor$ with probability $1-\varphi_{i}$ and $\omega_{i}=\left\lfloor w_{i}\right\rfloor+1$ with probability $\varphi_{i}$. (Note that Kuijvenhoven and Scholtus (2011) proposed to obtain these random inflation weights by applying Fellegi's method for controlled random rounding. This has the nice property that $\left|\hat{U}_{a}^{*}\right|=|U|$ holds with certainty, rather than just in expectation.)

2. For each $b=1, \ldots, B$ do the following:

a. Draw a sample $S_{a b}^{*}$ from $\hat{U}_{a}^{*}$ according to the same design that was used to draw $S$ from $U$. For $k \in \hat{U}_{a}^{*}$ the inclusion probability is chosen to be $\pi_{k}^{*} \propto \pi_{i}$, with $i$ the unit in the original sample $S$ of which unit $k$ is a copy. Here, the proportionality constant is chosen so that $\sum_{k \in \hat{U}_{a}^{*}} \pi_{k}^{*}=|S|$ holds.

b. Analogously to the original estimation procedure yielding $\hat{\theta}=t\left(S, U_{1}\right)$, construct the bootstrap replicate $\hat{\theta}_{a b}^{*}=t\left(S_{a b}^{*}, U_{1}\right)$.

3. Compute the variance estimate for $\hat{\theta}-\theta$ based on pseudo-population $\hat{U}_{a}^{*}$ as $v_{a}(\hat{\theta}-\theta)=(B-1)^{-1} \sum_{b=1}^{B}\left(\hat{\theta}_{a b}^{*}-\overline{\hat{\theta}_{a}^{*}}\right)^{2}$, with $\overline{\hat{\theta}_{a}^{*}}=B^{-1} \sum_{b=1}^{B} \hat{\theta}_{a b}^{*}$.

- Compute the final variance estimate for $\hat{\theta}-\theta$ by averaging over the pseudopopulations: $\widehat{\operatorname{var}}_{\text {boot }}(\hat{\theta}-\theta)=A^{-1} \sum_{b=1}^{A} v_{a}(\hat{\theta}-\theta)$.

The outer for loop of this algorithm is intended to reduce the noise due to the random assignment of integer-valued inflation weights to units with non-integer sampling weights in Step 1. Previous results in Chauvet (2007) and Kuijvenhoven and Scholtus (2011) suggest that this additional for loop may have little added value in practice (i.e., choosing $A=1$ leads to variance estimates of a similar accuracy as choosing $A>1$ ). It can 
certainly be avoided in the special case that all $w_{i}$ are integer-valued. For variance estimation, $B=200$ replicates are often considered sufficient in the bootstrap literature (Efron and Tibshirani 1993 sec. 6.4). If the sample $S$ is based on a multi-stage design, an extended version of the above pseudo-population approach may be used to account for clustering (Chauvet 2007; Mashreghi et al. 2016).

The contents of Step $2 b$ depend on the original estimation procedure. For $\hat{\theta}_{h c}$ in Equation (1). based on mass imputation, in this step we basically re-estimate the imputation model and use this to impute the missing values in the pseudo-population. In general, the bootstrap sample $S_{a b}^{*}$ may contain copies of units from $S \backslash S_{2}$, that is, units that overlap with the register part of the population. In analogy with the original imputation procedure, only the subset of units in $S_{a b}^{*}$ that originate from $S_{2}$, say $S_{2 a b}^{*}$, is used to reestimate the imputation model. Similarly, only the missing values for the subset of units in the pseudo-population $\hat{U}_{a}^{*}$ that originate from $S_{2}$, say $\hat{U}_{2 a}^{*}$, are subject to imputation. The missing values in $\hat{U}_{2 a}^{*}$ occur for those units that are not contained in the bootstrap sample $S_{2 a b}^{*}$, that is, $\hat{U}_{2 a}^{*} \backslash S_{2 a b}^{*}$. The register part of the pseudo-population is not imputed in the bootstrap procedure, as the contribution of the register part to $\hat{\theta}_{h c}$ is considered fixed. In summary, Step $2 b$ consists here of the following steps:

- Define $\hat{U}_{2 a}^{*}$ as the subpopulation of $\hat{U}_{a}^{*}$ consisting of copies of units from $S_{2}$, and define $S_{2 a b}^{*}=S_{a b}^{*} \cap \hat{U}_{2 a}^{*}$.

- Use $S_{2 a b}^{*}$ to re-estimate the imputation model for $y_{1}, \ldots, y_{C}$.

- Impute the missing values of $y_{1}, \ldots, y_{C}$ in $\hat{U}_{2 a}^{*} \backslash S_{2 a b}^{*}$ using the re-estimated model.

- Compute the replicate $\hat{\theta}_{h c, a b}^{*}=\sum_{k \in U_{1}} h_{k} y_{c k}+\left(\sum_{k \in S_{2 a b}^{*}} h_{k} y_{c k}+\sum_{k \in \hat{U}_{2 a b}^{*} S_{2 a b}^{*}} h_{k} \tilde{y}_{c k}\right)$ analogously to Equation (1).

Note that only the contribution of the non-register part to $\hat{\theta}_{h c, a b}^{*}$ varies between replicates.

The bootstrap method is straightforward to implement and can in fact re-use most of the code that was created to compute the original estimates. It is a computationally intensive method. A potentially useful aspect is that the time-consuming parts of the above bootstrap algorithm have to be performed only once. For instance, with mass imputation, the massimputed pseudo-populations could be stored and used to compute a variance estimate for any estimator $\hat{\theta}_{h c}$ by generating the replicates $\hat{\theta}_{h c, a b}^{*}$ 'on the fly'. Since each pseudopopulation consists of copies of units in the original sample $S$, all relevant information can be stored in a matrix of $|S| A B C$ integers; see Scholtus (2018) for details.

\section{The Continuation-Ratio Model for Imputation}

In Sections 5 and 6 we will present applications of the variance estimation methods of Sections 2 and 3 to simulated and real data on educational attainment. In these applications, use is made of the imputation approach proposed for educational attainment in the next Dutch virtual census. In the present section we will briefly discuss the underlying imputation model.

The imputation approach is based on logistic regression. Since educational attainment has $C>2$ categories, the binomial logistic regression model cannot be applied directly. To account for the fact that educational attainment is an ordinal variable, De Waal et al. 
(2018) proposed to use an extension of logistic regression known as the continuation-ratio model.

The continuation-ratio logistic regression model (Agresti $2013 \mathrm{sec}$. 8.3.6) consists of $C-1$ ordinary binomial logistic regression models. Each of these binomial models refers to the conditional probability $q_{c i}$ that person $i$ does not attain a higher level than a particular level $c$, given that this person at least reached level $c(c=1, \ldots, C-1)$ :

$$
\begin{aligned}
& q_{1 i}=P\left(y_{1 i}=1 \mid \mathbf{x}=\mathbf{x}_{i}\right) . \\
& q_{c i}=P\left(y_{c i}=1 \mid y_{1 i}=\ldots=y_{(c-1) i}=0, \mathbf{x}=\mathbf{x}_{i}\right), \quad(c=2, \ldots, C-1) .
\end{aligned}
$$

Here, $\mathbf{x}_{i}$ denotes a vector of auxiliary variables used in the model. Note that each conditional probability $q_{c i}$ refers to a binary choice $\left(y_{c i}=1\right.$ or $y_{c i}=0$ ). The continuationratio logistic regression model thus consists of a sequence of models of the form:

$$
\log \left(\frac{q_{c i}}{1-q_{c i}}\right)=\boldsymbol{\beta}_{c}^{T} \mathbf{x}_{i}, \quad(c=1, \ldots, C-1) .
$$

Agresti (2013) noted that maximum likelihood estimates of all parameters in the continuation-ratio model can be obtained by estimating the $C-1$ binomial logistic regression models in Equation (5) separately, each of them being estimated on the subset of the sample that satisfies the relevant condition of the form $y_{1 i}=\ldots=y_{(c-1) i}=0$. From the estimated model parameters, the conditional probability that a person with characteristics $\mathbf{x}_{i}$ has education level $c$ may then be predicted by

$$
\hat{q}_{c i}=\frac{\exp \left(\hat{\boldsymbol{\beta}}_{c}^{T} \mathbf{x}_{i}\right)}{1+\exp \left(\hat{\boldsymbol{\beta}}_{c}^{T} \mathbf{x}_{i}\right)}, \quad(c=1, \ldots, C-1) .
$$

Subsequently, predictions for the marginal probabilities $p_{c i}=P\left(y_{c i}=1 \mid \mathbf{x}=\mathbf{x}_{i}\right)$ as used in Section 2 can be derived by the following recursive relation:

$$
\begin{aligned}
& \hat{p}_{1 i}=\hat{q}_{1 i}, \\
& \hat{p}_{c i}=\hat{q}_{c i}\left(1-\sum_{k=1}^{c-1} \hat{p}_{k i}\right), \quad(c=2, \ldots, C-1), \\
& \hat{p}_{C i}=1-\sum_{c=1}^{C-1} \hat{p}_{c i} .
\end{aligned}
$$

To account for finite-population sampling - possibly with a complex survey design pseudo maximum likelihood estimation can be used (Chambers and Skinner 2003; see also Appendix, Subsection 8.2). Under this approach, a large-sample approximation to $\operatorname{cov}\left(\hat{p}_{c i}, \hat{p}_{c j}\right)$ for the predicted probabilities from the continuation-ratio model is derived in Appendix (Subsubsection 8.2.2). The computation of these approximate covariances involves a recursive algorithm over $c=1, \ldots, C$. Moreover, to evaluate Equations (3) or (4) this algorithm would need to be run for each pair $\left(i \in U_{2}, j \in U_{2}\right.$ ) or at least each pair with $h_{\mathrm{i}}=h_{\mathrm{j}}=1$, which can be computationally challenging for populations of realistic 
size. In practice, some efficiency may be gained by noting that $\operatorname{cov}\left(\hat{p}_{c i}, \hat{p}_{c j}\right)=\operatorname{cov}\left(\hat{p}_{c k}, \hat{p}_{c l}\right)$ whenever $\mathbf{x}_{\mathbf{i}}=\mathbf{x}_{\mathrm{k}}$ and $\mathbf{x}_{\mathbf{j}}=\mathbf{x}_{\mathbf{1}}$. (Scholtus 2018).

In general, Equation (2) need not hold exactly for the continuation-ratio model with $C>2$. Sufficient conditions under which Equation (2) holds exactly are provided in Appendix (Subsubsection 8.2.4). There is also argued that, for large samples, Equation (2) should hold approximately in practice provided that the imputation model contains $h$, the variables that define the sampling design of $S_{2}$, and the interaction of $h$ with these variables.

\section{Simulation Study}

An extensive test of the bootstrap method from Section 3 for various estimators based on weighting with combined register and survey data was conducted in Kuijvenhoven and Scholtus (2011). This bootstrap method has been in use at Statistics Netherlands since 2010 to estimate variances for publications on educational attainment based on weighting. In this section, we describe the results of a simulation study for an estimator based on mass imputation. We also compare the bootstrap variance estimates to the analytical estimates from Equation (4).

All computations were done in the $\mathrm{R}$ environment for statistical computing. The survey package (Lumley 2018) was used for pseudo maximum likelihood estimation. A fast implementation of the analytical variance estimator was created using the data.table package (Dowle et al. 2019).

As a basis for this study, we used the data of the synthetic Samplonia population (see, e.g., Bethlehem 2009). A target population of size $N=5 \times 745=3725$ was created by concatenating five copies of all persons aged over 14 in Samplonia. In this simulation, there were no register data, so $U_{1}=\varnothing$; and $U=U_{2}$. The sample $S=S_{2}$ was drawn according to a simple random sampling design without replacement, with sample size $n=\mathrm{N} / 5=745$.

Mass imputation of educational attainment for persons in $U_{2} \backslash S_{2}$ was based on a simplified version of the imputation approach proposed for the Dutch census, outlined in Section 4. In this simulation study, educational attainment was classified into $C=3$ categories, labelled as 'low', 'medium', and 'high'. The continuation-ratio model was applied with auxiliary information of the form gender $\times($ age + income $)$. Here, gender consisted of two classes, age consisted of three levels, and income was used as a continuous variable. This model was based on the available variables in the Samplonia data set; it should be noted that the imputation model proposed for the Dutch census uses different auxiliary variables (see Section 6).

The target frequency table in this study consisted of a cross-classification of age and educational attainment (both with three levels). Table 1 below shows the true population counts (left panel) and approximate true standard deviations of the mass-imputed Equation (1) for these counts (right panel). The latter were obtained by drawing 20,000 samples from the population and for each of them estimating the model, applying mass imputation and tabulating the target estimates.

Next, we simulated 100 samples from the population and estimated the variances by two approaches:

- using the analytical variance estimator (4), with $\widehat{\operatorname{cov}}\left(\hat{p}_{c i}, \hat{p}_{c j}\right)$ based on the largesample approximation in Appendix (Subsection 8.2); 
Table 1. True counts and simulated true standard deviations for an artificial population.

\begin{tabular}{lccccccr}
\hline & \multicolumn{3}{c}{ True counts } & & \multicolumn{2}{c}{ True standard deviations } \\
\hline & \multicolumn{2}{c}{ Educational attainment } & & \multicolumn{2}{c}{ Educational attainment } \\
\cline { 2 - 4 } Age (years) & Low & Medium & High & & Low & Medium & High \\
\hline Young (15-35) & 330 & 795 & 400 & & 34.5 & 42.2 & 36.8 \\
Middle (36-55) & 115 & 560 & 480 & & 22.3 & 36.8 & 36.1 \\
Old (56+) & 120 & 525 & 400 & & 22.8 & 35.6 & 34.5 \\
\hline
\end{tabular}

- using the bootstrap algorithm of Section 3, with $A=1$ (as no rounding was necessary here) and $B=200$.

Table 2 shows the mean estimated standard deviations and (in brackets) their standard deviation across 100 simulations. The table shows that both approaches produced estimated standard deviations that were close to their true values on average. From the values in brackets it can be seen that the estimated standard deviations from the analytical approach were more precise than those of the bootstrap approach (i.e., there was less variation between simulated samples).

We found that Equation (2) was approximately satisfied in these samples; this is as expected, since the imputation model included the variable age as a predictor and all observations had the same design weight. Finally, a note on computation times: across 100 simulated samples the average computation time was about 2.4 minutes per sample for the bootstrap method and just under five seconds per sample for the analytical method.

\section{Application to Real Data}

\subsection{Setup}

In this application we estimate variances for one table for the Dutch Population and Housing Census 2011. The table under consideration contains the Dutch population by Geographic area (12 categories), Sex (two categories), Age (eight categories) and Educational attainment (six categories), which makes up $12 \times 2 \times 8 \times 6=1,152$ cells in

Table 2. Mean and standard deviation of estimated standard deviations for estimated counts after mass imputation, based on 100 simulations.

\begin{tabular}{|c|c|c|c|c|c|c|}
\hline \multirow[b]{3}{*}{ Age (years) } & \multicolumn{3}{|c|}{ Estimated analytical st. dev. } & \multicolumn{3}{|c|}{ Estimated bootstrap st. dev. } \\
\hline & \multicolumn{3}{|c|}{ Educational attainment } & \multicolumn{3}{|c|}{ Educational attainment } \\
\hline & Low & Medium & High & Low & Medium & High \\
\hline Young (15-35) & $\begin{array}{c}34.1 \\
(1.3)\end{array}$ & $\begin{array}{c}41.8 \\
(0.7)\end{array}$ & $\begin{array}{c}36.6 \\
(1.2)\end{array}$ & $\begin{array}{l}34.1 \\
(2.2)\end{array}$ & $\begin{array}{l}41.9 \\
(2.3)\end{array}$ & $\begin{array}{c}36.4 \\
(2.0)\end{array}$ \\
\hline Middle (36-55) & $\begin{array}{l}22.5 \\
(1.7)\end{array}$ & $\begin{array}{c}36.8 \\
(0.8)\end{array}$ & $\begin{array}{c}36.1 \\
(1.0)\end{array}$ & $\begin{array}{l}22.7 \\
(2.4)\end{array}$ & $\begin{array}{l}36.6 \\
(2.0)\end{array}$ & $\begin{array}{c}36.0 \\
(2.1)\end{array}$ \\
\hline Old $(56+)$ & $\begin{array}{c}22.8 \\
(1.8)\end{array}$ & $\begin{array}{c}35.4 \\
(0.8)\end{array}$ & $\begin{array}{c}34.3 \\
(1.0)\end{array}$ & $\begin{array}{l}22.5 \\
(1.9)\end{array}$ & $\begin{array}{c}35.2 \\
(2.1)\end{array}$ & $\begin{array}{c}34.5 \\
(2.2)\end{array}$ \\
\hline
\end{tabular}


total. Geographic area, Sex and Age are available from central population registers that fully cover the target population of the census. Educational attainment is available for a subset of the population.

In this evaluation study Educational attainment is estimated from the Educational Attainment File (EAF), with reference day January 1, 2011. The EAF is a database that includes data from multiple registers and LFS data from multiple years. Data from the EAF can be matched to the population registers at the micro level. The EAF is planned to be used for the 2021 census.

The EAF contains a 'register part' and a 'non-register part', which include 9,363,909 and $7,291,890$ persons, respectively. These two parts refer to the people for which register information on educational attainment is and is not available $\left(U_{1}\right.$ and $U_{2}$ in the notation of Section 2). The focus of this simulation study is entirely on the non-register part. For 340,472 out of 7,291,890 persons, educational levels are available from an LFS. The missing observations are imputed at the micro level, using the continuation-ratio model that was introduced in Section 4 . The imputed data are used to estimate counts and variances for the aforementioned table: Geographic area $\times$ Sex $\times$ Age $\times$ Educational attainment.

It should be noted that for this study we had only limited information about the origin of the sample data $S_{2}$. First, we did not have any information about the larger sample $S$ from which $S_{2}$ was obtained by removing the overlap with $U_{1}$. Second, the data in $S_{2}$ are an integrated sample of several LFS rounds and we only had the final recalibrated sampling weights but no information about the underlying sampling design and the way different years were combined. For the purpose of this study, we approximated the design of $S_{2}$ by that of a simple random sample without replacement from $U_{2}$. This should give reasonable results for comparing the analytical and bootstrap methods, as the same approximation was used for both approaches. For a future application to the real Dutch census, a better approximation will be made which accounts for the complex survey design of the LFS.

The EAF-based data set was enriched with information from other data sources that are included in the system of Social Statistical Data sets (SSD). The variable Income (six categories: five quantiles and unknown/not available) was used as a stratification variable in the imputation model; that is to say, a separate continuation-ratio model was estimated for each income class. Income has been chosen because it has a relatively strong association with Educational attainment (Daalmans 2017). The variables Age, Geographic area and Sex that are contained in the target table were also used as an auxiliary variable for most of the cases. However, for the lowest class of education and three strata of income only Sex was taken as auxiliary variable. This choice will be explained further on.

As before, the bootstrap method in Section 3 was implemented with $B=200$ and $A=1$ and all computations were done using $\mathrm{R}$ and the survey and data.table packages.

\subsection{Results}

The computation time was much longer for the bootstrap method than for the analytical method: about 21.5 hours versus 48 minutes and 26 seconds. Note that the bootstrap computations could easily be parallelised across multiple processors to save time. However, we did not do this here. 
For the analytical Equation (4), for each cell of the target table a double sum needs to be computed of all people that have the same combination of values for the auxiliary variables in the imputation model, that is, Age, Sex and Geographic area and stratification variable Income. The maximum number of items over which this sum extends was 2,937 and the average number was 296 . The largest double sum contained approximately nine million elements $\left(2937^{2}\right)$, which does not seem too problematic from a computational point of view.

A first question is whether 200 bootstrap iterations are enough. To answer that question, we consider the ratio of the estimated standard deviations after 200 and 190 iterations. Table 3 below shows percentiles of these ratios for the 1,152 cells of the target table

For all but a few cells the difference is smaller than $\pm 3 \%$. For more than half of the cells the difference is even below $\pm 1 \%$. These results also show that there is no structural upward or downward trend of the standard deviations between the 190th and 200th iteration.

We now give an impression about the results of the bootstrap procedure. The coefficient of variation $(\mathrm{CV})$ has been computed for each cell, that is, the ratio of the estimated standard deviation to the mean, that is, the average cell count over the 200 bootstrap samples. As we consider the non-register part of the EAF only, the mean is derived from the non-register part of the EAF.

In Figure 1, the CV is plotted against the sample size of the cell. As expected, cells with the least number of sample survey observations have the highest CVs.

We now turn to the main question of this simulation exercise: the difference in results for the standard deviations between the bootstrap method and the analytical approximation. First, we plot the CVs of both approaches against each other in Figure 2, subdivided for each (imputed) educational category.

The graph shows similarities between the CVs of both methods, especially for the categories 3 and 4 that are the most frequently occurring. To assess this further, percentiles of the ratios of the CVs are given in Table 4. These ratios compare the CVs of the analytical method with those of the bootstrap method and have been derived from all cells, that is, all educational levels.

The difference between the analytical and the bootstrap method is reasonably small. For instance, the median value is 0.97 , the 90 th percentile is 1.79 and the 10 th percentile is 0.59. This gives us some empirical evidence that the analytical procedure gives close approximations to the true variance.

In Figure 3, density plots are shown of the ratio of the CVs of the analytical and bootstrap method. The solid black line represents the density of all cells in the table. The other lines represent the density for subsets of $20 \%$ of cells in the table, where the cells are ordered by sample size from smallest (quintile 1) to largest (quintile 5). As expected, the largest deviations occur for cells with relatively few observations. Interestingly, it appears that the analytical method tends to underestimate the CV slightly compared to the bootstrap method for all quintiles except the first one, where it has a tendency to overestimate the $\mathrm{CV}$.

Table 3. Percentile of the ratios of standard deviations after 200 and 190 iterations.

\begin{tabular}{llllllllll}
\hline Percentile & 0.01 & 0.05 & 0.10 & 0.25 & 0.50 & 0.75 & 0.90 & 0.95 & 0.99 \\
SD-ratio & 0.981 & 0.985 & 0.987 & 0.992 & 0.998 & 1.007 & 1.014 & 1.020 & 1.035 \\
\hline
\end{tabular}




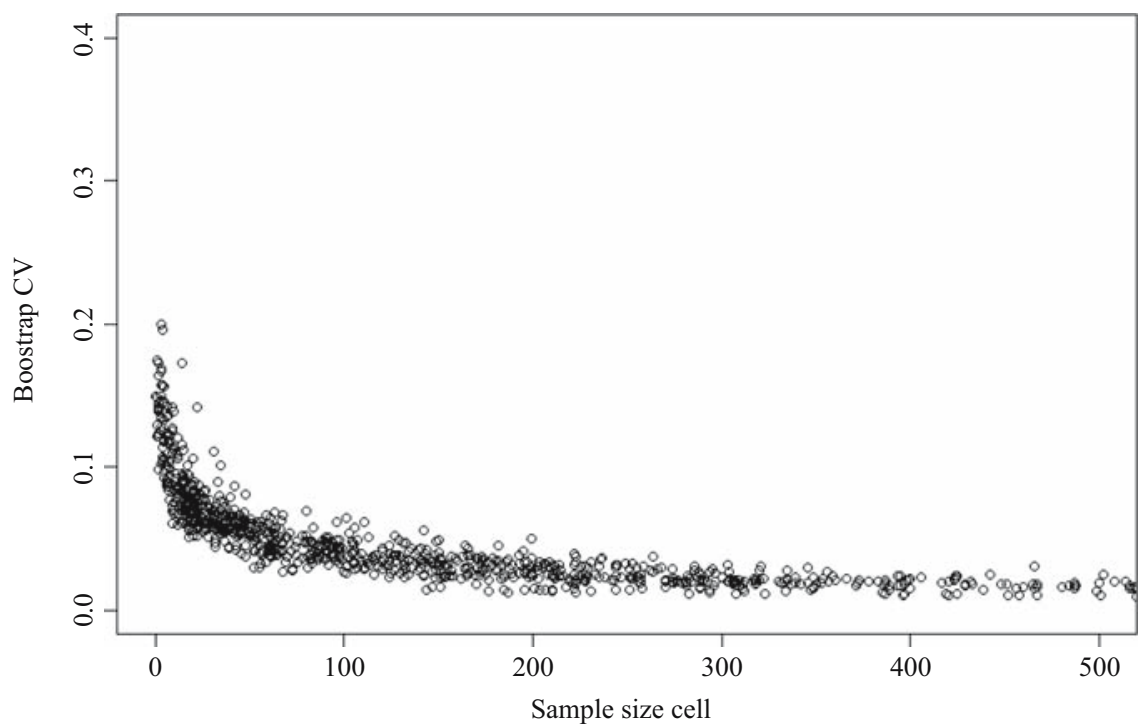

Fig. 1. Coefficient of variation for the bootstrap method.

A possible explanation for this underestimation in cells where the sample size is sufficiently large is that the condition in Equation (2) does not hold in this application, as the imputation model contains the main effects of Sex, Geographic area and Age but not their interactions. Thus, variance Equation (4) neglects a source of uncertainty; see also the discussion at the end of Appendix (Subsection 8.1). To illustrate this further, we also
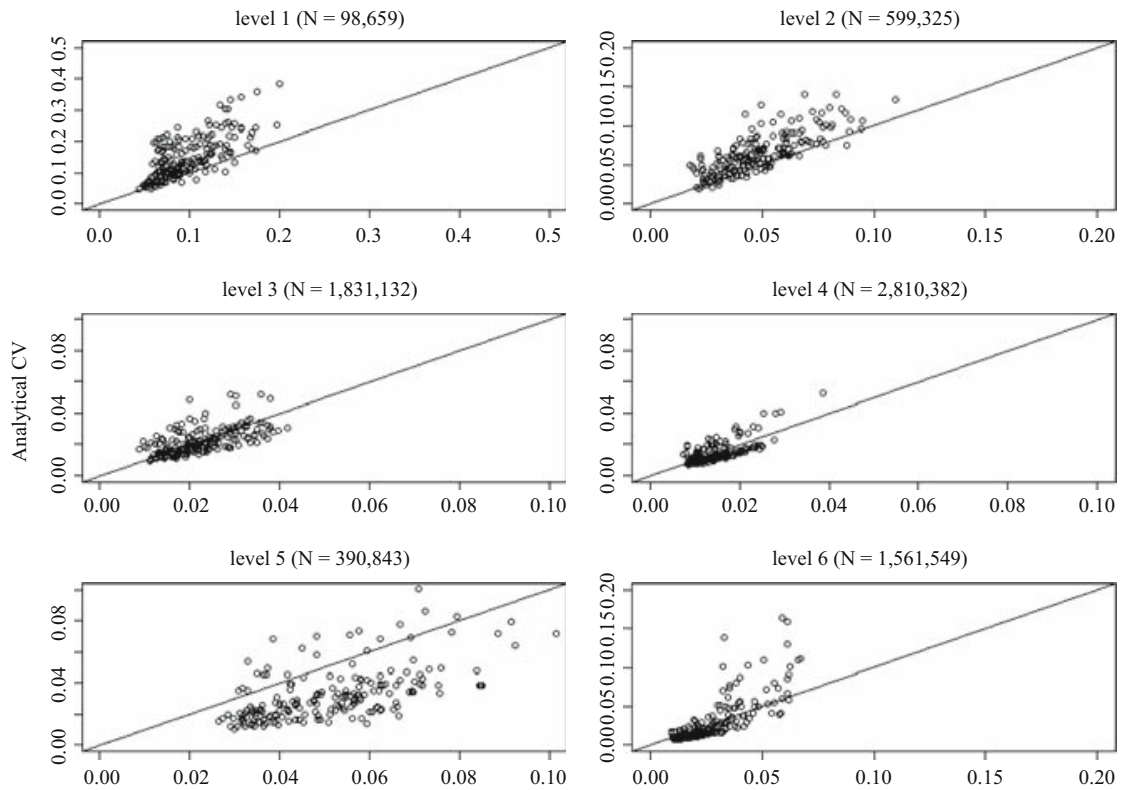

Bootstrap CV

Fig. 2. Coefficient of variation; analytical method against bootstrap. 
Table 4. Percentiles of the ratios of CVs based on the analytical and bootstrap method.

\begin{tabular}{llllllllll}
\hline Percentiles & 0.01 & 0.05 & 0.10 & 0.25 & 0.50 & 0.75 & 0.90 & 0.95 & 0.99 \\
CV-ratios & 0.356 & 0.494 & 0.589 & 0.748 & 0.967 & 1.360 & 1.785 & 2.086 & 2.760 \\
\hline
\end{tabular}

computed the analytical CVs and bootstrap CVs for the table Geographic area $\times$ Educational attainment, that is, one of the marginal tables of the original table. For this lower-dimensional table, Equation (2) is expected to hold approximately in this application. In Figure 4, the ratio of the CVs of the analytical and bootstrap method is plotted against the sample size per cell for the 72 cells in this table. It is seen that here the two approaches are in closer agreement and that the ratio of the CVs tends to one for cells with large sample sizes.

Finally, it should be noted that for some strata the number of observations was too small for a reliable estimation of all regression coefficients. Initially, Sex, Geographic area and Age were included in the model for each stratum. For some strata, this led to extremely high analytically derived standard deviations, when compared to the bootstrap. The problem was especially apparent for the highest income classes. In these strata, low educational attainment barely occurs. As a consequence, the regression coefficients for estimating the probability of low educational levels could not be reliably estimated. Due to near-multicollinearity of the auxiliary variables, extremely high standard deviations were returned for some coefficients by Equation (9) in Appendix (Subsubsection 8.2.1). The impact of this diminished after reducing the regression model, that is, after using only Sex as an auxiliary variable in certain strata. This shows that the analytical approach is sensitive to model selection. It also appears that the analytical variance estimates are more sensitive to model over-parametrisation than the mass-imputed estimates themselves.

Model selection is an important step in an application to the census anyhow, due to the availability of a large number of auxiliary variables. All variables that appear in the target

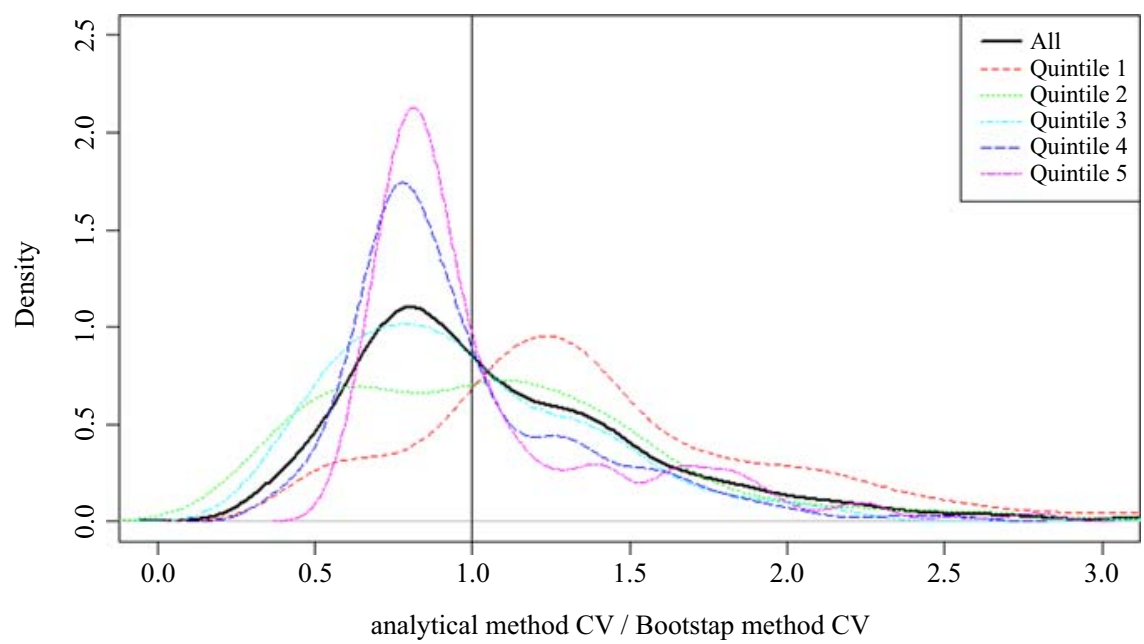

Fig. 3. Ratio of coefficient of variation according to the analytical and bootstrap methods; density of all cells and density by quintiles based on sample size per cell. 


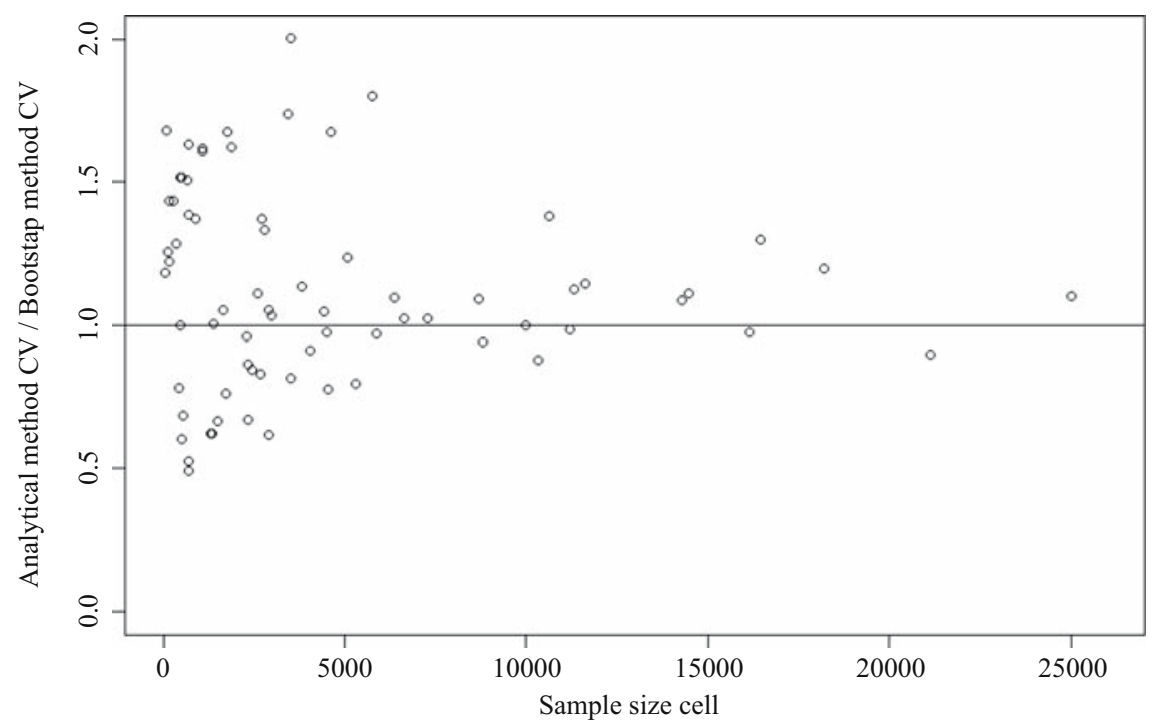

Fig. 4. Ratio of coefficient of variation according to the analytical and bootstrap methods (marginal table: geographic area $\times$ educational attainment).

tables to be produced can be used. These are: Age, Country of citizenship, Place of usual residence, Household status, Industry / sector of economic activity, Size of locality, Place of birth, Place of usual residence one year prior to the census, Sex, Status in employment, and Year of arrival in the country. Some of these have a large number of categories, which often include 'rare' cases. An example from the Dutch census is the category "Oceanic" for Country of citizenship. On the one hand, it is attractive to include as many auxiliary variables in the imputation model as available, because a relation between a target variable (to be imputed) and some other variable can only be properly measured if that other variable is taken as an auxiliary variable in the imputation model. Also, a model with few auxiliary variables is more likely to be mis-specified, leading to bias in the mass-imputed estimator. On the other hand, as we have seen before, including many auxiliary variables in the imputation model might lead to a large variance, because the number of observations can be insufficient for reliable estimation of the regression coefficients. Hence, a trade-off must be made between variance and (potential) bias and it can be inevitable to aggregate some categories of auxiliary variables in an imputation model. Several automatic procedures can be implemented for this purpose. In the analytical approach, one might manually 'optimise' the aggregation level of the auxiliary variables for the sample survey at hand. In the bootstrap approach, one must take into account that different resamples are obtained at each iteration. Therefore, the bootstrap methods require some automation in the selection of the imputation model.

\section{Discussion and Conclusion}

This article has developed a new design-based variance formula for estimated frequency tables after mass imputation of one of the variables. A case is considered in which one target variable is observed for a sample of the population and where all missing values for the target population need to be imputed. Furthermore, it is postulated that the imputations 
are based on a model that predicts, for each record, the probabilities for the different categories of the target variable.

The above problem is relevant for the Dutch census. One of the census variables, educational attainment, cannot be observed for the entire target population. All nonobserved values can be imputed, for instance by using logistic regression or one of its variants. Several frequency tables need to be produced for the Dutch census that crossclassify educational attainment with other variables that are observed for the whole population. The analytical expressions in this article can be used to estimate the variances of the cells of these tables. Although the case of the Dutch census has been mentioned often in this article, the problem is more generally relevant for other applications in which a categorical variable is imputed for an entire target population.

In Section 2 we presented an analytical variance expression that allows for a broad class of imputation methods. Each imputation method gives rise to a specific completion of the expression. The special case of the continuation-ratio model was considered in Section 4. We have derived a new analytical variance approximation for this specific model.

As an illustration, the analytical approximation was applied to a Dutch census table in Section 6. The results have been compared to outcomes of a bootstrap method, adapted from Kuijvenhoven and Scholtus (2011), which can be applied more generally to estimators based on combined data sources. The results demonstrated that the analytical variance approximation works reasonably well. For the large majority of cases, the estimated standard deviation was between 0.5 and 1.5 times the standard error of the bootstrap method. Moreover, we have demonstrated that both methods can be applied to a data set of a significant size, that is, approximately seven million imputations.

The analytical variance estimator was derived under the simplifying assumption that Equation (2) holds, at least approximately. This condition can be verified in practice: if the left-hand-side of Equation (2) is small in comparison to $\hat{\theta}_{h c 2}$, then the analytical variance approximation proposed here should work well. In situations where this condition does not hold, our analytical approach is likely to underestimate the true variance for cells with a sufficiently large sample size (see Appendix, Subsection 8.1). The question of the designbased variance of the mass-imputed Equation (1) is relevant for our statistical office and, we believe, for the wider official statistical community. As far as we are aware, there is no analytical method currently available in the literature to derive this variance in a general setting, which avoids introducing Equation (2) or some other assumption that might be violated in practice. Alternatively, the bootstrap method from Section 3 may be used regardless of whether Equation (2) holds.

In general, the computation time can be a limiting factor for the estimation of variances for large data sets. The analytical approximation requires the computation of a double sum. The number of terms in this sum can become quite large. However, the bootstrap method can be expected to have an even larger computational burden for many applications. The bootstrap method requires work that is equivalent to imputing the entire target population many times. In our case study, approximately seven million records needed to be imputed 200 times. This led to a computation time that was about a factor 30 larger than for the analytical approximation. This illustrates that, although the bootstrap is 
more flexible than the analytical approach, analytical approximations may still be useful and even necessary for variance estimation in practice.

Our application also revealed that the analytical approximation of the variances is more sensitive to model over-parametrisation than the mass-imputed estimates themselves. This stresses the importance of using the analytical approximation in combination with a proper model selection method. When this is neglected, there is a risk of a serious over-estimation of variances.

An alternative variance estimation approach that was not considered in this study is multiple imputation (Rubin 1987). Similar to our bootstrap method, this would require imputing the missing values of educational attainment throughout the population several times. In fact, the bootstrap algorithm of Section 3 could be adapted to be used for multiple imputation. A practical advantage of multiple imputation compared to direct bootstrapping would be that it requires fewer replicates. A limitation of multiple imputation, compared to the approaches considered here, is that it cannot be used to estimate the variance of an arbitrary estimator, but only that of the associated multiple imputation estimator. Thus, in this approach the variance estimation method actually guides the choice of the estimator itself, which then has to be based on a multiply-imputed file. For practical reasons there are currently no plans to generate more than one imputation per person in the official microdata of the Dutch virtual census, so multiple imputation is not an option for variance estimation in this application. For this reason, it was not considered here. However, it may be interesting to compare multiple imputation to our analytical and bootstrap method in a more extensive future study.

Future work may focus on extending the variance estimation methods considered here. The analytical approach could be extended to the case of imputing multiple categorical variables, or a combination of categorical and numerical variables. An extension of the analytical approach is also needed to handle imputation methods that are not based on a parametric model, such as hot deck imputation. Finally, it may be interesting to develop variance estimation techniques - either analytical or by means of resampling - that can account for uncertainty in the measurement of register-based variables, including the effects of micro-integration (Bakker 2011) when overlapping data are available from a register and a survey.

\section{Appendix}

\subsection{Derivation of Variance Formula (3)}

Conditioning on a realisation of the random sample $S_{2}$ and using a standard decomposition formula for conditional variances, we can write the variance of the estimator $\hat{\theta}_{h c}$ in Equation (1) as:

$$
\operatorname{var}\left(\hat{\theta}_{h c}-\theta_{h c}\right)=E\left\{\operatorname{var}\left(\hat{\theta}_{h c}-\theta_{h c} \mid S_{2}\right)\right\}+\operatorname{var}\left\{E\left(\hat{\theta}_{h c}-\theta_{h c} \mid S_{2}\right)\right\} .
$$

Substituting the definitions of $\theta_{h c}$ and $\hat{\theta}_{h c}$ from Equation (1) and using the fact that imputations for different persons are independent, we obtain (with $\theta_{h c 2}=\sum_{i \in U_{2}} h_{i}$ $\left.y_{c i}\right)$ : 


$$
\begin{aligned}
\operatorname{var}\left(\hat{\theta}_{h c}-\theta_{h c}\right)= & E\left\{\operatorname{var}\left(\hat{\theta}_{h c 2}-\theta_{h c 2} \mid S_{2}\right)\right\}+\operatorname{var}\left\{E\left(\hat{\theta}_{h c 2}-\theta_{h c 2} \mid S_{2}\right)\right\} \\
= & E\left\{\operatorname{var}\left(\sum_{i \in U_{2} \backslash S_{2}} h_{i}\left(\tilde{y}_{c i}-y_{c i}\right) \mid S_{2}\right)\right\} \\
& +\operatorname{var}\left\{E\left(\sum_{i \in U_{2} \backslash S_{2}} h_{i}\left(\tilde{y}_{c i}-y_{c i}\right) \mid S_{2}\right)\right\} \\
= & E\left\{\sum_{i \in U_{2} \backslash S_{2}} h_{i} \operatorname{var}\left(\tilde{y}_{c i}-y_{c i} \mid S_{2}\right)\right\}+\operatorname{var}\left\{\sum_{i \in U_{2} \backslash S_{2}} h_{i} E\left(\tilde{y}_{c i}-y_{c i} \mid S_{2}\right)\right\} \\
= & E\left\{\sum_{i \in U_{2} \backslash S_{2}} h_{i} \hat{p}_{c i}\left(1-\hat{p}_{c i}\right)\right\}+\operatorname{var}\left\{\sum_{i \in U_{2} \backslash S_{2}} h_{i}\left(\hat{p}_{c i}-y_{c i}\right)\right\} .
\end{aligned}
$$

For the third equality it was used that $h_{i}^{2}=h_{i}$. For the last equality it was used that $E\left(\tilde{y}_{c i} \mid S_{2}\right)=\hat{p}_{c i}$ and $\operatorname{var}\left(\tilde{y}_{c i} \mid S_{2}\right)=\hat{p}_{c i}\left(1-\hat{p}_{c i}\right)$ based on a multinomial distribution. The first term in this expression is easily estimated in practice (cf. Equation (4)), so we leave this term as it is and focus on elaborating the second term.

It should be noted that $\hat{p}_{c i}$, the predicted probability that person $i$ belongs to category $c$, is obtained from a model that is estimated on the observed data from sample $S_{2}$. Hence, $\hat{p}_{c i}$ is a random variable that depends on $S_{2}$. In general, this complicates the evaluation of the above variance component. However, under the assumption that Equation (2) holds, the second term can be simplified and we find:

$$
\begin{aligned}
\operatorname{var}\left\{\sum_{i \in U_{2} \backslash S_{2}} h_{i}\left(\hat{p}_{c i}-y_{c i}\right)\right\} & =\operatorname{var}\left\{\sum_{i \in U_{2}} h_{i}\left(\hat{p}_{c i}-y_{c i}\right)\right\} \\
& =\sum_{i \in U_{2}} \sum_{j \in U_{2}} h_{i} h_{j} \operatorname{cov}\left(\hat{p}_{c i}-y_{c i}, \hat{p}_{c j}-y_{c i}\right) \\
& =\sum_{i \in U_{2}} \sum_{j \in U_{2}} h_{i} h_{j} \operatorname{cov}\left(\hat{p}_{c i}, \hat{p}_{c j}\right) .
\end{aligned}
$$

The last equality follows since we treat the values of $y_{c i}$ in the finite population as fixed here. Hence, Equation (3) is obtained.

More generally, if Equation (2) is not satisfied, it follows from the above derivation that

$$
\operatorname{var}\left(\hat{\theta}_{h c}-\theta_{h c}\right)=\tilde{V}+V_{S_{2}}-2 C_{U_{2}, S_{2}},
$$

where $\tilde{V}$ denotes the variance from Equation (3), $V_{S_{2}}=\operatorname{var}\left\{\sum_{i \in S_{2}} h_{i}\left(\hat{p}_{c i}-y_{c i}\right)\right\}$ and $C_{U_{2}, S_{2}}=\operatorname{cov}\left\{\sum_{i \in U_{2}} h_{i}\left(\hat{p}_{c i}-y_{c i}\right), \sum_{i \in S_{2}} h_{i}\left(\hat{p}_{c i}-y_{c i}\right)\right\}$. Since $\sum_{i \in S_{2}} h_{i}\left(\hat{p}_{c i}-y_{c i}\right)$ is an unweighted sample total, it seems intuitively reasonable to expect the contributions of the terms $V_{S_{2}}$ and $C_{U_{2}, S_{2}}$ to $\operatorname{var}\left(\hat{\theta}_{h c}-\theta_{h c}\right)$ to be negligible if the sampling fraction of $S_{2}$ is 'small enough', even when Equation (2) does not hold. 
To make this argument somewhat more precise, we suppose that $S_{2}$ is a simple random sample with $\left|S_{2}\right| /\left|U_{2}\right| \ll 1$. Let $N_{h}$ denote the number of units in $U_{2}$ with $h_{i}=1$ and let $n_{h}$ denote the expected number of units in $S_{2}$ with $h_{i}=1$. It can be shown that the standard Horvitz-Thompson estimator for $\theta_{h c}$ then has a variance of order $O\left(N_{h}^{2} / n_{h}\right)$. In practice, the available background variables to impute $y_{c}$ usually have limited predictive value, so we expect the variance of the mass-imputed estimator to have the same order of magnitude: $\operatorname{var}\left(\hat{\theta}_{h c}-\theta_{h c}\right)=O\left(N_{h}^{2} / n_{h}\right)$. (We have also confirmed this empirically for the application in Section 6; results not shown here.) Furthermore, if Equation (2) does not hold, $V_{S_{2}}$ has the same order as

$$
\operatorname{var}\left\{\sum_{i \in S_{2}} h_{i}\left(p_{c i}-y_{c i}\right)\right\}=n_{h}^{2} \operatorname{var}\left\{\frac{1}{n_{h}} \sum_{i \in S_{2}} h_{i}\left(p_{c i}-y_{c i}\right)\right\}=O\left(n_{h}\right) .
$$

Here, we used that $\hat{p}_{c i}-p_{c i}$ is of a smaller order than $p_{c i}-y_{c i}=O(1)$ and that $\operatorname{var}\left\{\frac{1}{n_{h}} \sum_{i \in S_{2}} h_{i}\left(p_{c i}-y_{c i}\right)\right\}=O\left(1 / n_{h}\right)$; see Särndal et al. $(1992,214)$ for the latter result. For the covariance term, we find:

$$
\left|C_{U_{2}, S_{2}}\right| \leq \sum_{i \in U_{2}} h_{i} \sqrt{\operatorname{var}\left(\hat{p}_{c i}-y_{c i}\right)} \sqrt{V_{S_{2}}}=O\left(N_{h} \sqrt{n_{h}}\right) .
$$

Thus, if $S_{2}$ is a simple random sample and $n_{h} \ll N_{h}^{2 / 3}$, then both $V_{S_{2}}$ and $C_{U_{2}, S_{2}}$ are of negligible order and it follows that $\operatorname{var}\left(\hat{\theta}_{h c}-\theta_{h c}\right) \approx \tilde{V}$ even when Equation (2) does not hold exactly.

Furthermore, under the weaker condition $n_{h} \ll N_{h}$ the term $V_{S_{2}}$ is still negligible but $C_{U_{2}, S_{2}}$ may not be when Equation (2) does not hold. Note that

$$
C_{U_{2}, S_{2}}=V_{S_{2}}+\operatorname{cov}\left\{\sum_{i \in U_{2} \backslash S_{2}} h_{i}\left(\hat{p}_{c i}-y_{c i}\right), \sum_{i \in S_{2}} h_{i}\left(\hat{p}_{c i}-y_{c i}\right)\right\} .
$$

Provided that the imputation model does not introduce bias, the average prediction error $h_{i}\left(\hat{p}_{c i}-y_{c i}\right)$ over $U_{2}$ should tend to cancel out for repeated samples. This suggests that we may expect in practice that $\operatorname{cov}\left\{\sum_{i \in U_{2} \backslash S_{2}} h_{i}\left(\hat{p}_{c i}-y_{c i}\right), \sum_{i \in S_{2}}\right.$ $\left.h_{i}\left(\hat{p}_{c i}-y_{c i}\right)\right\}<0$. Therefore, since

$$
\operatorname{var}\left(\hat{\theta}_{h c}-\theta_{h c}\right)=\tilde{V}-V_{S_{2}}-2 \operatorname{cov}\left\{\sum_{i \in U_{2} \backslash S_{2}} h_{i}\left(\hat{p}_{c i}-y_{c i}\right), \sum_{i \in S_{2}} h_{i}\left(\hat{p}_{c i}-y_{c i}\right)\right\}
$$

for cells where $V_{S_{2}}$ is negligible but $C_{U_{2}, S_{2}}$ is not, we may expect that $\operatorname{var}\left(\hat{\theta}_{h c}-\theta_{h c}\right)>\tilde{V}$. That is to say, when Equation (2) is violated and $n_{h} \ll N_{h}$ but not $n_{h} \ll N_{h}^{2 / 3}$, then Equation (3) is more likely to underestimate the true variance than to overestimate it.

Although these conclusions have been derived for the case of a simple random sample, we expect that they extend to other sampling designs that are commonly used in practice for social surveys. In practice, the available design variables for social surveys usually are correlated only weakly to the variables of interest, leading to a limited design effect on the order of magnitude of various variance terms. 


\subsection{Details of the Continuation-Ratio Model}

\subsection{1. (Pseudo) Maximum Likelihood Theory for the Continuation-Ratio Model}

First, suppose that $S_{2}$ were a sample of independent, identically distributed observations from the underlying distribution of the continuation-ratio model of Section 4. Agresti (2013, sec. 8.3.6) shows that the log likelihood function may then be written as:

$$
\ell=\sum_{c=1}^{C-1} \sum_{i \in S_{2, \geq c}}\left\{y_{c i}\left(\boldsymbol{\beta}_{c}^{T} \mathbf{x}_{i}\right)-\log \left[1+\exp \left(\boldsymbol{\beta}_{c}^{T} \mathbf{x}_{i}\right)\right]\right\}
$$

where $S_{2, \geq 1}=S_{2}$ and $S_{2, \geq c}(c=2, \ldots, C-1)$ denotes the subsample of units in $S_{2}$ with $y_{1 i}=\ldots=y_{(c-1) i}=0$. As the $C-1$ terms of $\ell$ have no parameters in common, it is clear that maximum likelihood estimates can be obtained by solving the following likelihood equations:

$$
\begin{aligned}
0 & =\frac{\partial \ell}{\partial \boldsymbol{\beta}_{c}}=\sum_{i \in S_{2, \geq c}}\left\{y_{c i} \mathbf{x}_{i}-\frac{\exp \left(\hat{\boldsymbol{\beta}}_{c}^{T} \mathbf{x}_{i}\right)}{1+\exp \left(\hat{\boldsymbol{\beta}}_{c}^{T} \mathbf{x}_{i}\right)} \mathbf{x}_{i}\right\} \\
& =\sum_{i \in S_{2, \geq c}}\left(y_{c i}-\hat{q}_{c i}\right) \mathbf{x}_{i}(c=1, \ldots, C-1)
\end{aligned}
$$

where the last equality follows from Equation (6). Clearly, this is equivalent to fitting each binomial logistic regression model in Equation (5) separately to the associated subsample $S_{2, \geq c}$. Furthermore, it follows from standard inference theory for maximum likelihood that, asymptotically, for any pair of vectors $\hat{\boldsymbol{\beta}}_{c}$ and $\hat{\boldsymbol{\beta}}_{d}(c \neq d)$,

$$
\left(\begin{array}{l}
\hat{\boldsymbol{\beta}}_{c}-\boldsymbol{\beta}_{c} \\
\hat{\boldsymbol{\beta}}_{d}-\boldsymbol{\beta}_{d}
\end{array}\right) \sim N\left(\left(\begin{array}{c}
\mathbf{0} \\
\mathbf{0}
\end{array}\right),\left(\begin{array}{cc}
\left\{X^{T} \Delta_{\geq c} X\right\}^{-1} & 0 \\
0 & \left\{X^{T} \Delta_{\geq d} X\right\}^{-1}
\end{array}\right)\right)
$$

here, the matrix $X$ contains rows $\mathbf{x}_{i}^{T}$ for each unit and $\Delta_{\geq_{c}}$ denotes an associated diagonal matrix with $\left(\Delta_{\geq 1}\right)_{i i}=q_{1 i}\left(1-q_{1 i}\right)$ and, for $c>1$,

$$
\left(\Delta_{\geq c}\right)_{i i}=\left\{\begin{array}{cl}
q_{c i}\left(1-q_{c i}\right) & \text { if } y_{1 i}=\ldots=y_{(c-1) i}=0 \\
0 & \text { otherwise. }
\end{array}\right.
$$

For a more detailed derivation, see Scholtus (2018).

In a finite-population setting, instead of the above approach pseudo maximum likelihood estimation can be used (Chambers and Skinner 2003 chap. 2). The original model is then interpreted as a superpopulation model from which the target population has been generated. The parameters of interest (say, $\boldsymbol{\beta}_{U_{2}, c}$ ) are now implicitly defined by the likelihood equations that would be obtained if $y_{c i}$ were observed for the entire population 
$U_{2}$ :

$$
\begin{aligned}
0 & =\sum_{i \in U_{2, \geq c}}\left\{y_{c i} \mathbf{x}_{i}-\frac{\exp \left(\boldsymbol{\beta}_{U_{2}, c}^{T} \mathbf{x}_{i}\right)}{1+\exp \left(\boldsymbol{\beta}_{U_{2}, c}^{T} \mathbf{x}_{i}\right)} \mathbf{x}_{i}\right\} \\
& =\sum_{i \in U_{2, \geq c}}\left(y_{c i}-q_{U_{2}, c i}\right) \mathbf{x}_{i} \quad(c=1, \ldots, C-1),
\end{aligned}
$$

where $U_{2, \geq_{c}}$ is defined analogously to $S_{2, \geq_{c}}$. Note that here we ignore the register part of the population $U_{1}$ as there is no need to estimate an imputation model for these units.

To account for the sample design of $S_{2}$, the parameters $\boldsymbol{\beta}_{U_{2}, c}$ are estimated from a weighted version of the likelihood equations:

$$
\begin{aligned}
0 & =\sum_{i \in S_{2, \geq c}} w_{2 i}\left\{y_{c i} \mathbf{x}_{i}-\frac{\exp \left(\hat{\boldsymbol{\beta}}_{c}^{T} \mathbf{x}_{i}\right)}{1+\exp \left(\hat{\boldsymbol{\beta}}_{c}^{T} \mathbf{x}_{i}\right)} \mathbf{x}_{i}\right\} \\
& =\sum_{i \in S_{2, \geq c}} w_{2 i}\left(y_{c i}-\hat{q}_{c i}\right) \mathbf{x}_{i}(c=1, \ldots, C-1),
\end{aligned}
$$

with $w_{2 i}=1 / \pi_{2 i}$ the design weight of unit $i \in S_{2}$ based on its inclusion probability $\pi_{2 i}$. (Note: if $S_{2}$ is actually a subsample of a larger sample $S$ from $U$, as discussed in Section 2 , then these inclusion probabilities should also reflect the subsampling step.) This provides consistent estimates of $\boldsymbol{\beta}_{U_{2}, c}$ (and $\boldsymbol{\beta}_{c}$ ).

It follows from a Taylor series linearisation (Chambers and Skinner 2003, 23) that the large-sample variance of the estimated parameters with respect to the finite population can be estimated by the following 'sandwich' estimator:

$$
\widehat{\operatorname{var}}\left(\hat{\boldsymbol{\beta}}_{c}-\boldsymbol{\beta}_{U_{2}, c}\right)=\left(X^{T} \hat{\Delta}_{\geq c, w_{2}} X\right)^{-1} \hat{\Gamma}_{c}\left(X^{T} \hat{\Delta}_{\geq c, w_{2}} X\right)^{-1} .
$$

Here, $\hat{\Delta}_{\geq c, w_{2}}$ is a diagonal matrix with elements $\left(\hat{\Delta}_{\geq 1, w_{2}}\right)=w_{2 i} \hat{q}_{1 i}\left(1-\hat{q}_{1 i}\right)$ and, for $c>1$,

$$
\left(\hat{\Delta}_{\geq c, w_{2}}\right)_{i i}=\left\{\begin{array}{cl}
w_{2 i} \hat{q}_{c i}\left(1-\hat{q}_{c i}\right) & \text { if } y_{1 i}=\ldots=y_{(c-1) i}=0 \\
0 & \text { otherwise, }
\end{array}\right\}
$$

while $\hat{\Gamma}_{c}$ is a design-based estimator of $\operatorname{var}\left(\sum_{i \in S_{2, \geq c}} w_{2 i}\left(y_{c i}-q_{U_{2}, c i}\right) \mathbf{x}_{i}\right)$. Since the latter expression is an ordinary Horvitz-Thompson estimator, we can use (Särndal et al. 1992, 48):

$$
\hat{\Gamma}_{c}=\sum_{i \in S_{2, \geq c}} \sum_{j \in S_{2, \geq c}} \frac{\pi_{2 i j}-\pi_{2 i} \pi_{2 j}}{\pi_{2 i j}} \mathbf{x}_{i}\left(y_{c i}-\hat{q}_{c i}\right)\left(y_{c j}-\hat{q}_{c j}\right) \mathbf{x}_{j}^{T},
$$

where $\pi_{2 i j}$ denotes a second-order inclusion probability of $S_{2}$. Finally, by a similar argument it can be shown that $\hat{\boldsymbol{\beta}}_{c}-\boldsymbol{\beta}_{U_{2}, c}$ and $\hat{\boldsymbol{\beta}}_{d}-\boldsymbol{\beta}_{U_{2}, d}(c \neq d)$ are still asymptotically independent. 


\subsubsection{Conditional Probabilities: Large-Sample Covariances}

Given the results in Subsubsection 8.2.1, large-sample variances and covariances of the predicted conditional probabilities $\hat{q}_{c i}$ can be derived in a straightforward manner from a first-order Taylor series approximation to Equation (6). For pseudo maximum likelihood estimation it follows that, asymptotically, $\operatorname{cov}\left(\hat{q}_{c i}, \hat{q}_{d j}\right) \approx 0$ for $c \neq d$, while $\operatorname{cov}\left(q_{c i}, q_{c j}\right)$ is estimated consistently by:

$$
\widehat{\operatorname{cov}}\left(\hat{q}_{c i}, \hat{q}_{c j}\right)=\hat{q}_{c i}\left(1-\hat{q}_{c i}\right) \hat{q}_{c j}\left(1-\hat{q}_{c j}\right) \mathbf{x}_{i}^{T}\left(X^{T} \hat{\Delta}_{\geq c, w_{2}} X\right)^{-1} \hat{\Gamma}_{c}\left(X^{T} \hat{\Delta}_{\geq c, w_{2}} X\right)^{-1} \mathbf{x}_{j}
$$

In particular, it follows that predicted conditional probabilities for different levels of educational attainment are asymptotically independent. We refer to Scholtus (2018) for more details on the derivation.

\subsubsection{Marginal Probabilities: Large-Sample Covariances}

Before proceeding to the marginal probabilities $\hat{p}_{c i}$, we introduce the following useful lemmas.

- Lemma 1. For any set of four random variables $A, B, C$ and $D$ such that

- $A$ is independent of $B$ and $D$, and

- $C$ is independent of $B$ and $D$,

it holds that $\operatorname{cov}(A B, C D)=E(A C) \operatorname{cov}(B, D)+E(B) E(D) \operatorname{cov}(A, C)$.

- Lemma 2. For any set of three random variables $X, Y$ and $Z$ such that $X$ is independent of $Y$ and $Z$, it holds that $\operatorname{cov}(X Y, Z)=E(X) \operatorname{cov}(Y, Z)$.

Lemma 1 may be derived by conditioning on $A$ and $C$. Lemma 2 follows as a corollary of the first lemma by choosing $A=X, B=Y, C=1$ and $D=Z$.

Denote $\operatorname{cov}\left(\hat{p}_{c i}, \hat{p}_{d j}\right)=C_{c d i j}$, for $i, j \in U_{2}$ and $1 \leq c, d \leq C$. To evaluate the second component of variance formula (3), we only need the 'diagonal' terms $C_{c c i j}$. However, to derive expressions for these diagonal terms it will be seen below that we also need to consider the terms $C_{c d i j}$ with $c \neq d$.

Since $\hat{p}_{1 i}=\hat{q}_{1 i}, \quad C_{11 i j}=\operatorname{cov}\left(\hat{q}_{1 i}, \hat{q}_{1 j}\right)$ can be estimated by Equation (10). For $2 \leq c \leq C-1$, using Equation (7) we can write:

$$
C_{c c i j}=\operatorname{cov}\left\{\hat{q}_{c i}\left(1-\sum_{k=1}^{c-1} \hat{p}_{k i}\right), \hat{q}_{c j}\left(1-\sum_{l=1}^{c-1} \hat{p}_{l j}\right)\right\} .
$$

According to Equation (7), each $\hat{p}_{k i}$ is constructed from just the probabilities $\hat{q}_{1 i}, \ldots, \hat{q}_{k i}$. Since the conditional probabilities $\hat{q}_{1 i}, \ldots, \hat{q}_{(C-1) i}$ are known to be asymptotically mutually independent, it follows that $\hat{q}_{c i}$ is asymptotically independent of all $\hat{p}_{1 i}, \ldots, \hat{p}_{(c-1) i}$ and all $\hat{p}_{1 j}, \ldots, \hat{p}_{(c-1) j}$ for $j \neq i$. Therefore, we can (asymptotically) apply Lemma 1 to the above expression for $C_{c c i j}$, with $A=\hat{q}_{c i}$, 
$B=1-\sum_{k=1}^{c-1} \hat{p}_{k i}, C=\hat{q}_{c j}$ and $D=1-\sum_{l=1}^{c-1} \hat{p}_{l j}$. This yields:

$$
\begin{aligned}
C_{c c i j} \approx & E\left(\hat{q}_{c i} \hat{q}_{c j}\right) \operatorname{cov}\left(1-\sum_{k=1}^{c-1} \hat{p}_{k i}, 1-\sum_{l=1}^{c-1} \hat{p}_{l j}\right) \\
& +E\left(1-\sum_{k=1}^{c-1} \hat{p}_{k i}\right) E\left(1-\sum_{l=1}^{c-1} \hat{p}_{l j}\right) \operatorname{cov}\left(\hat{q}_{c i}, \hat{q}_{c j}\right),
\end{aligned}
$$

and therefore (for $c=2, \ldots, C-1$ )

$$
\begin{aligned}
C_{c c i j} \approx & \left\{\operatorname{cov}\left(\hat{q}_{c i}, \hat{q}_{c j}\right)+q_{c i} q_{c j}\right\} T_{c-1, i j} \\
& +\left(1-\sum_{k=1}^{c-1} p_{k i}\right)\left(1-\sum_{l=1}^{c-1} p_{l j}\right) \operatorname{cov}\left(\hat{q}_{c i}, \hat{q}_{c j}\right),
\end{aligned}
$$

with the short-hand notation

$$
T_{c, i j}=\sum_{k=1}^{c} \sum_{l=1}^{c} C_{k l i j}, \quad(c=1, \ldots, C-1)
$$

(Note: in a finite-population context, in Equation (11) $q_{c i}$ and $p_{c i}$ should be replaced by $q_{U_{2}, c i}$ and the associated marginal probabilities $p_{U_{2}, c i}$. In what follows we will ignore this distinction as it is not essential to the argument). For the remaining case $c=C$, it follows directly from Equation (7) that

$$
C_{C C i j}=\operatorname{cov}\left(1-\sum_{k=1}^{C-1} \hat{p}_{k i}, 1-\sum_{l=1}^{C-1} \hat{p}_{l j}\right)=\sum_{k=1}^{C-1} \sum_{l=1}^{C-1} \operatorname{cov}\left(\hat{p}_{k i}, \hat{p}_{l j}\right)=T_{C-1, i j} .
$$

It remains to find an expression for $T_{c, i j}$. The following recursion is derived at the end of this subsection: for $c=1, \ldots, C-1$ it holds asymptotically that

$$
T_{c, i j} \approx \sum_{k=1}^{c} C_{k k i j}-\sum_{k=2}^{c}\left(q_{k i}+q_{k j}\right) T_{k-1, i j}
$$

with the convention that the second sum is zero for $c=1$.

We now have all the required ingredients to estimate all terms $\operatorname{cov}\left(\hat{p}_{c i}, \hat{p}_{c j}\right)=C_{c c i j}$ that occur in Equation (3), at least for large samples. The following algorithm can be used:

1. Estimate $\operatorname{cov}\left(\hat{q}_{c i}, \hat{q}_{c j}\right)$ by (10) for all $c=1, \ldots, C-1$ and define $\hat{C}_{11 i j}=\hat{T}_{1, i j}=\widehat{\operatorname{cov}}\left(\hat{q}_{1 i}, \hat{q}_{1 j}\right)$.

2. Repeat the following steps for $c=2, \ldots, C-1$ :

a. Estimate $C_{c c i j}$ in line with (11) by

$$
\hat{C}_{c c i j}=\left\{\widehat{\operatorname{cov}}\left(\hat{q}_{c i}, \hat{q}_{c j}\right)+\hat{q}_{c i} \hat{q}_{c j}\right\} \hat{T}_{c-1, i j}+\left(1-\sum_{k=1}^{c-1} \hat{p}_{k i}\right)\left(1-\sum_{l=1}^{c-1} \hat{p}_{l j}\right) \widehat{\operatorname{cov}}\left(\hat{q}_{c i}, \hat{q}_{c j}\right) .
$$


b. Estimate $T_{c, i j}$ in line with Equation (13) by

$$
\hat{T}_{c, i j}=\sum_{k=1}^{c} \hat{C}_{k k i j}-\sum_{k=2}^{c}\left(\hat{q}_{k i}+\hat{q}_{k j}\right) \hat{T}_{k-1, i j} .
$$

3. Finally, define $\hat{C}_{C C i j}=\hat{T}_{C-1, i j}$ in line with Equation (12).

An illustration of the first steps of this algorithm is given in Scholtus (2018). Note that the algorithm manages to avoid a circular argument, because $C_{c c i j}$ is estimated in Step 2a using $\hat{T}_{c-1, i j}$ and $T_{c, i j}$ is estimated in Step $2 \mathrm{~b}$ using only the estimated covariances $\hat{C}_{11 i j}, \ldots, \hat{C}_{c c i j}$.

We conclude this subsection by deriving expression (13). By definition, the expression holds exactly for $c=1$. Therefore, suppose that $2 \leq c \leq C-1$. We begin by evaluating the 'off-diagonal' terms $C_{c d i j}$ with $c \neq d$. First suppose that $d<c$. We can write:

$$
C_{c d i j}=\operatorname{cov}\left\{\hat{q}_{c i}\left(1-\sum_{k=1}^{c-1} \hat{p}_{k i}\right), \hat{p}_{d j}\right\} \text {. }
$$

Since $\hat{q}_{c i}$ is asymptotically independent of all $\hat{p}_{1 i}, \ldots, \hat{p}_{(c-1) i}$ and also of $\hat{p}_{d j}$, Lemma 2 can be applied to this expression, with $X=\hat{q}_{c i}, Y=1-\sum_{k=1}^{c-1} \hat{p}_{k i}$ and $Z=\hat{p}_{d j}$. This yields:

$$
C_{c d i j} \approx E\left(\hat{q}_{c i}\right) \operatorname{cov}\left(1-\sum_{k=1}^{c-1} \hat{p}_{k i}, \hat{p}_{d j}\right)=-q_{c i} \sum_{k=1}^{c-1} C_{k d i j}, \quad(d<c)
$$

Similarly, we obtain for $d<c$ that $C_{d c i j} \approx-q_{c j} \sum_{l=1}^{c-1} C_{d l i j}$.

These expressions can be substituted in the definition of $T_{c, i j}$, to find:

$$
\begin{aligned}
T_{c, i j} & =\sum_{k=1}^{c}\left\{C_{k k i j}+\sum_{k-1}^{l=1} C_{k l i j}+\sum_{l=k+1}^{c} C_{k l i j}\right\} \\
& \approx \sum_{k=1}^{c}\left\{C_{k k i j}-\sum_{l=1}^{k-1}\left(q_{k i} \sum_{m=1}^{k-1} C_{m l i j}\right)-\sum_{l=k+1}^{c}\left(q_{l j} \sum_{m=1}^{l-1} C_{k m i j}\right)\right\} \\
& =\sum_{k=1}^{c} C_{k k i j}-\sum_{k=2}^{c}\left(q_{k i} \sum_{l=1}^{k-1} \sum_{m=1}^{k-1} C_{m l i j}\right)-\sum_{l=2}^{c}\left(q_{l j} \sum_{k=1}^{l-1} \sum_{m=1}^{l-1} C_{k m i j}\right) \\
& =\sum_{k=1}^{c} C_{k k i j}-\sum_{k=2}^{c} q_{k i} T_{k-1, i j}-\sum_{l=2}^{c} q_{l j} T_{l-1, i j},
\end{aligned}
$$

from which Equation (13) follows. In the third line, we used that the middle term is empty (hence zero) for $k=1$ and we re-arranged the summation over $k$ and $l$ in the right-most term.

\subsubsection{Marginal Probabilities: Equation (2)}

Suppose first that the imputation model satisfies the following Assumptions: 
1. The model includes the cross-classified variable $h$ as a predictor.

2. The model includes all variables that determine the sampling design of $S_{2}$ as predictors.

3. All predictor variables are categorical (coded as dummy variables) and the model contains all higher-order interactions of these predictor variables.

The following lemma establishes in particular that, under these Assumptions, Equation (2) is satisfied exactly by the predicted marginal probabilities $\hat{p}_{c i}$ from the continuation-ratio model.

Lemma 3. Under Assumptions 2 and 3 it holds that $\sum_{i \in S_{2}} \mathbf{x}_{i} \hat{p}_{c i}=\sum_{i \in S_{2}} \mathbf{x}_{i} y_{c i}$ for $c=1, \ldots, C$.

Proof. First, it follows from Equation (8) that in this case, for all $c=1, \ldots, C-1$,

$$
\sum_{i \in S_{2, \geq c}} \mathbf{x}_{i} \hat{q}_{c i}=\sum_{i \in S_{2, \geq c}} w_{2 i}\left(\mathbf{x}_{i} \pi_{2 i}\right) \hat{q}_{c i}=\sum_{i \in S_{2, \geq c}} w_{2 i}\left(\mathbf{x}_{i} \pi_{2 i}\right) y_{c i}=\sum_{i \in S_{2, \geq c}} \mathbf{x}_{i} y_{c i}=\sum_{i \in S_{2}} \mathbf{x}_{i} y_{c i}
$$

The second equality follows because, under Assumptions 2 and $3, \mathbf{x}_{i} \pi_{2 i}$ is contained in the span of $\mathbf{x}_{i}$; the last equality follows because $y_{c i}=0$ for all $i \in S_{2} \backslash S_{2, \geq c}$. In particular, this establishes that $\sum_{i \in S_{2}} \mathbf{x}_{i} \hat{p}_{1 i}=\sum_{i \in S_{2}} \mathbf{x}_{i} y_{1 i}$ since $\hat{p}_{1 i}=\hat{q}_{1 i}$.

Now suppose that $\sum_{i \in S_{2}} \mathbf{x}_{i} \hat{p}_{k i}=\sum_{i \in S_{2}} \mathbf{x}_{i} y_{k i}$ has been shown to hold for all $k=1, \ldots, c-1$. For $\hat{p}_{c i}$ (with $c=2, \ldots, C-1$ ) we find from Equation (7):

$$
\begin{aligned}
\sum_{i \in S_{2}} \mathbf{x}_{i} \hat{p}_{c i} & =\sum_{i \in S_{2}} \mathbf{x}_{i} \hat{q}_{c i}\left(1-\sum_{k=1}^{c-1} \hat{p}_{k i}\right)=\sum_{i \in S_{2}} \mathbf{x}_{i} \hat{q}_{c i}\left(1-\sum_{k=1}^{c-1} y_{k i}+\sum_{k=1}^{c-1} y_{k i}-\sum_{k=1}^{c-1} \hat{p}_{k i}\right) \\
& =\sum_{i \in S_{2, \geq c}} \mathbf{x}_{i} \hat{q}_{c i}-\sum_{i \in S_{2}} \mathbf{x}_{i} \hat{q}_{c i}\left\{\sum_{k=1}^{c-1}\left(\hat{p}_{k i}-y_{k i}\right)\right\} \\
& =\sum_{i \in S_{2}} \mathbf{x}_{i} y_{c i}-\sum_{k=1}^{c-1} \sum_{i \in S_{2}} \mathbf{x}_{i} \hat{q}_{c i}\left(\hat{p}_{k i}-y_{k i}\right) .
\end{aligned}
$$

Thus, to establish the result for $\hat{p}_{c i}$, it suffices to show that $\sum_{i \in S_{2}} \mathbf{x}_{i} \hat{q}_{c i}\left(\hat{p}_{k i}-y_{k i}\right)=0$ for all $k=1, \ldots, c-1$. Consider an infinite Taylor series expansion of $\hat{q}_{c i}$ from Equation (6). in terms of $\hat{\boldsymbol{\beta}}_{c}^{T} \mathbf{x}_{i}$ around 0. Each term in this series contains a product of one or more elements of the vector $\mathbf{x}_{i}$. By assumption 3 above, all of these products are contained in $\mathbf{x}_{i}$ itself. One further application of assumption 3 yields that $\mathbf{x}_{i} \hat{q}_{c i}$ is also contained in the span of $\mathbf{x}_{i}$. Hence, it follows by induction that $\sum_{i \in S_{2}} \mathbf{x}_{i} \hat{q}_{c i}\left(\hat{p}_{k i}-y_{k i}\right)=0$ for all $k=$ $1, \ldots, c-1$ and that $\sum_{i \in S_{2}} \mathbf{x}_{i} \hat{p}_{c i}=\sum_{i \in S_{2}} \mathbf{x}_{i} y_{c i}$.

Finally, to complete the proof of Lemma 3, for $c=C$ we find from Equation (7) that

$$
\sum_{i \in S_{2}} \mathbf{x}_{i} \hat{p}_{C i}=\sum_{i \in S_{2}} \mathbf{x}_{i}\left(1-\sum_{k=1}^{C-1} \hat{p}_{k i}\right)=\sum_{i \in S_{2}} \mathbf{x}_{i}\left(1-\sum_{k=1}^{C-1} y_{k i}\right)=\sum_{i \in S_{2}} \mathbf{x}_{i} y_{C i}
$$

In practice, Equation (3) will often not be satisfied. Higher-order interaction terms may be excluded from the imputation model if they are not significant, and there may also be 
predictor variables on a continuous scale. Consider the following weaker version of Equation (3):

$3^{*}$ ) The model includes the interaction of $h$ and the variables of the sampling design.

We argue that, for large samples and provided that Assumptions 2 and $3^{*}$ are satisfied, Equation (2) should still hold approximately.

Namely, under Assumptions 2 and $3 *$ it follows analogously to Equation (8) that $\sum_{i \in S_{2}} h_{i} \hat{p}_{c i}^{m l r}=\sum_{i \in S_{2}} h_{i} y_{c i}$ for predicted probabilities $\hat{p}_{c i}^{m l r}$ based on an ordinary multinomial logistic regression model. Furthermore, Agresti (2013, 301) notes that, compared to this multinomial logistic regression model, the main benefits of using a specialised model such as continuation-ratio that reflects the ordinal aspect of the target variable are "improved model parsimony and power" in small samples. If the sample is large enough, the ordinary multinomial logistic regression model should be able to capture the ordinal aspect nearly as well. This suggests that, asymptotically, it should hold that $\sum_{i \in S_{2}} h_{i} \hat{p}_{c i} / \sum_{i \in S_{2}} h_{i} \hat{p}_{c i}^{m l r} \rightarrow 1$. Hence, when Equation (2) holds exactly in the multinomial logistic regression model, it should also hold approximately in the continuation-ratio model.

\section{References}

Agresti, A. 2013. Categorical Data Analysis (Third Edition). New York: John Wiley and Sons.

Bakker, B.F.M. 2011. "Micro-integration: State of the Art." In ESSnet on Data Integration, Report on WP1: 77-107. Available at: http://ec.europa.eu/eurostat/cros/ content/essnet-di-final-report-wp1_en (accessed October 2020).

Bethlehem, J. 2008. "Surveys without Questions." In International Handbook of Survey Methodology, edited by E.D. de Leeuw, J.J. Hox, and D.A. Dillman: 500-511. New York: Psychology Press.

Bethlehem, J. 2009. Applied Survey Methods: A Statistical Perspective. Hoboken, NJ: John Wiley and Sons.

Booth, J.G., R.W. Butler, and P. Hall. 1994. "Bootstrap Methods for Finite Populations." Journal of the American Statistical Association 89: 1282-1289. DOI: http://doi.org/10. 1080/01621459.1994.10476868.

Canty, A.J., and A.C. Davison. 1999. "Resampling-based Variance Estimation for Labour Force Surveys.” The Statistician 48: 379-391. DOI: http://doi.org/10.1111/1467-9884. 00196.

Chambers, R.L., and C.J. Skinner, eds. 2003. Analysis of Survey Data. Chicester: John Wiley and Sons.

Chauvet, G. 2007. Méthodes de Bootstrap en Population Finie. PhD Thesis, Rennes: ENSAI. Available at: http://pastel.archives-ouvertes.fr/tel-00267689/document. (accessed October 2020).

Daalmans, J. 2017. Mass Imputation for Census Estimation. Discussion Paper, The Hague: Statistics Netherlands. Available at: http://www.cbs.nl/en-gb/background/2017/11/ mass-imputation-for-census-estimation. (accessed October 2020). 
Daalmans, J. 2018. "Divide-and-Conquer Solutions for Estimating Large Consistent Table Sets." Statistical Journal of the IAOS 34: 223-233. DOI: http://doi.org/10.3233/ SJI-170375.

De Waal, T., J. Daalmans, and F. Linder. 2018. Mass Imputation for Census Estimation: Methodology. Report, The Hague: Statistics Netherlands. Available at: http://ec.europa. eu/eurostat/cros/system/files/admin_wp6_2016_nl.pdf (accessed October 2020).

De Waal, T., J. Pannekoek, and S. Scholtus. 2011. Handbook of Statistical Data Editing and Imputation. Hoboken, NJ: John Wiley and Sons.

Dowle, M., et al. 2019. data.table: Extension of data.frame. R package version 1.12.0. Available at: http://cran.R-project.org/package = data.table. (accessed October 2020).

Efron, B. 1979. "Bootstrap Methods: Another Look at the Jackknife." The Annals of Statistics 7: 1-26. DOI: http://doi.org/10.1214/aos/1176344552.

Efron, B., and R.J. Tibshirani. 1993. An Introduction to the Bootstrap. London: Chapman \& Hall/CRC.

Gross, S.T. 1980. "Median Estimation in Sample Surveys.” In Proceedings of the Section on Survey Research Methods: American Statistical Association, August 11-14, 1980: 181-184. Houston, Texas: American Statistical Association. Available at: http://www. asasrms.org/Proceedings/papers/1980_037.pdf (accessed October 2020).

Kim, J.K., S. Park, Y. Chen, and C. Wu. 2020. "Combining Non-probability and Probability Survey Samples Through Mass Imputation.” Unpublished manuscript. Available at: https://arxiv.org/abs/1812.10694 (accessed October 2020).

Knottnerus, P., and C. van Duin. 2006. "Variances in Repeated Weighting with an Application to the Dutch Labour Force Survey." Journal of Official Statistics 22: 565-584. Available at: https://www.scb.se/contentassets/ca21efb41fee47d293bbee $5 \mathrm{bf} 7 \mathrm{be} 7 \mathrm{fb} 3 /$ variances-in-repeated-weighting-with-an-application-to-the-dutchlabour-force-survey.pdf (accessed March 2021).

Kuijvenhoven, L., and S. Scholtus. 2011. Bootstrapping Combined Estimators based on Register and Sample Survey Data. Discussion Paper, The Hague: Statistics Netherlands. Available at: http://www.cbs.nl/nl-nl/achtergrond/2011/39/bootstrapping-combined-estimator-based-on-register-and-sample-survey-data. (accessed October 2020).

Lumley, T. 2018. survey: Analysis of Complex Survey Samples. R package version 3.35. Available at: http://cran.R-project.org/package = survey. (accessed October 2020).

Mashreghi, Z., D. Haziza, and C. Léger. 2016. "A Survey of Bootstrap Methods in Finite Population Sampling." Statistics Surveys 10: 1-52. DOI: http://doi.org/10.1214/ 16-SS113.

Rubin, D.B. 1987. Multiple Imputation for Nonresponse in Surveys. New York: John Wiley and Sons.

Särndal, C.-E., B. Swensson, and J. Wretman. 1992. Model Assisted Survey Sampling. New York: Springer-Verlag.

Scholtus, S. 2018. Variances of Census Tables after Mass Imputation of Educational Attainment. Discussion Paper, The Hague: Statistics Netherlands. Available at: http:// www.cbs.nl/en-gb/background/2018/49/variances-of-census-tables-after-mass-imputation. (accessed October 2020).

Schulte Nordholt, E., M. Hartgers, and R. Gircour, eds. 2004. The Dutch Virtual Census of 2001. Analysis and Methodology. Voorburg/Heerlen: Statistics Netherlands. Available 
at: http://www.cbs.nl/en-gb/publication/2005/43/the-dutch-virtual-census-of-2001. (accessed October 2020).

Schulte Nordholt, E., J. van Zeijl, and L. Hoeksma (eds.). 2014. Dutch Census 2011. Analysis and Methodology. The Hague/Heerlen: Statistics Netherlands. Available at: http://www.cbs.nl/en-gb/publication/2014/47/dutch-census-2011 (accessed October 2020).

Valliant, R., A.H. Dorfman, and R.M. Royall. 2000. Finite Population Sampling and Inference: A Prediction Approach. New York: John Wiley and Sons.

Received May 2019

Revised January 2020

Accepted October 2020 